\title{
Manipulation of polarization anisotropy in bare InAs and InAs/GaSb core-shell nanowires
}

Cite as: Appl. Phys. Lett. 112, 153104 (2018); https://doi.org/10.1063/1.5021714

Submitted: 08 January 2018 . Accepted: 30 March 2018. Published Online: 11 April 2018

Atanu Patra, Anushree Roy, Umesh Prasad Gomes, Valentina Zannier, Daniele Ercolani, and Lucia Sorba
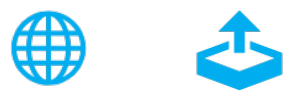

View Online

Export Citation

\section{ARTICLES YOU MAY BE INTERESTED IN}

Microstructure and interface analysis of emerging $\mathrm{Ga}(\mathrm{Sb}, \mathrm{Bi})$ epilayers and $\mathrm{Ga}(\mathrm{Sb}, \mathrm{Bi}) / \mathrm{GaSb}$ quantum wells for optoelectronic applications

Applied Physics Letters 112, 151905 (2018); https://doi.org/10.1063/1.5024199

Crystal-phase intergradation in InAs nanostructures grown by van der Waals heteroepitaxy on graphene

Applied Physics Letters 112, 142101 (2018); https://doi.org/10.1063/1.5017251

In-situ laser nano-patterning for ordered InAs/GaAs(001) quantum dot growth

Applied Physics Letters 112, 153108 (2018); https://doi.org/10.1063/1.5016096

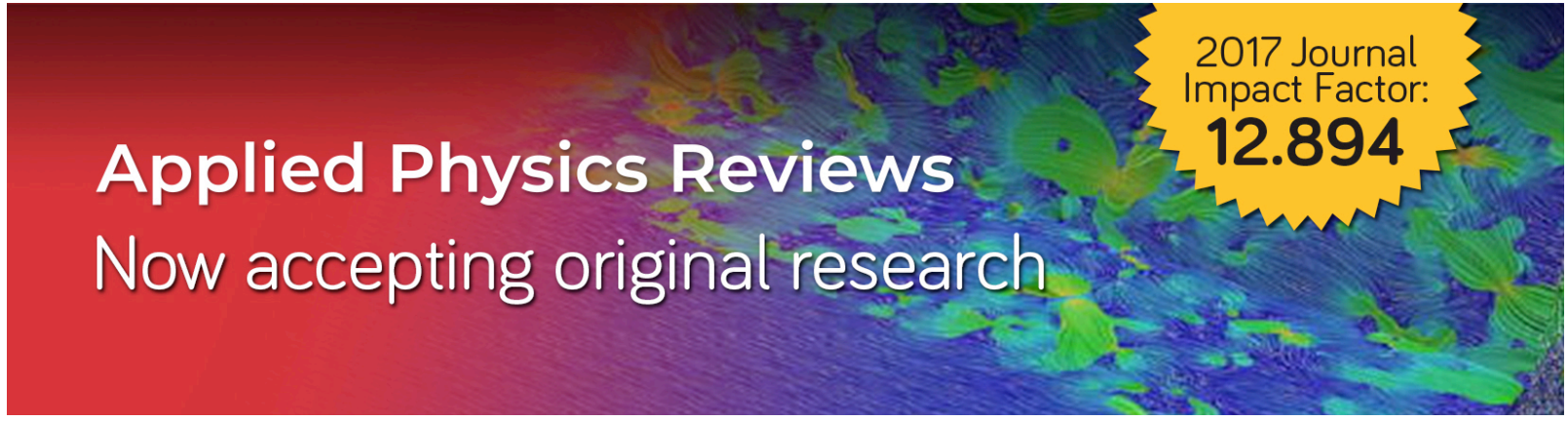




\title{
Manipulation of polarization anisotropy in bare InAs and InAs/GaSb core-shell nanowires
}

\author{
Atanu Patra, ${ }^{1}$ Anushree Roy, ${ }^{1, a)}$ Umesh Prasad Gomes, ${ }^{2}$ Valentina Zannier, ${ }^{2}$ \\ Daniele Ercolani, ${ }^{2}$ and Lucia Sorba ${ }^{2, b)}$ \\ ${ }^{1}$ Department of Physics, Indian Institute of Technology Kharagpur, Kharagpur 721 302, India \\ ${ }^{2}$ NEST-Istituto Nanoscienze-CNR and Scuola Normale Superiore, Piazza S. Silvestro 12, I-56127 Pisa, Italy
}

(Received 8 January 2018; accepted 30 March 2018; published online 11 April 2018)

\begin{abstract}
In this article, we compare the excitation wavelength dependence of the polarization anisotropy $(\rho)$ of an internal field induced Raman scattering signal in individual bare InAs and InAs/GaSb coreshell nanowires. The measured value of $\rho$ of the Raman scattering intensity for InAs/GaSb coreshell nanowires has a minimum at $\sim 500 \mathrm{~nm}$, while for the bare InAs nanowire, the value of $\rho$ monotonically increases over the same range of wavelengths. We have modeled the scattering intensities of both systems by considering the joint role of Raman tensor components and confinement of electromagnetic radiation inside the nanowire at two orthogonal polarization configurations of the electromagnetic radiation. The theoretical results allow us to understand that the observed behavior of $\rho$ is related to the nanowire geometry and to the difference in the wavelength dependence of the dielectric constants of InAs and GaSb. This work shows the possibility of manipulating the polarization anisotropy by selecting suitable diameters and materials for the core and the shell of the nanowire. We also report a six-fold increase in Raman scattering intensity due to the GaSb shell on InAs nanowires. Published by AIP Publishing. https://doi.org/10.1063/1.5021714
\end{abstract}

In recent times, the novel electronic and optical properties of III-V semiconductor nanowires (NWs) have drawn special attention because of their potential applications as nanoscale building blocks in optoelectronic device fabrication. Fabrication of core-shell nanostructures is in need as the shell over the core reduces the surface states and hence increases the efficiency of the devices. ${ }^{1,2}$ The studies on the polarization anisotropy $(\rho)$ of absorption, emission, or scattering of electromagnetic (EM) radiation in these systems demand special attention in view of their possible use as electromagnetically coupled semiconductor nanostructures in polarization-sensitive infra-red photonic devices. ${ }^{3}$ Although the efficacy of core-shell III-V semiconductor NWs as tunnelling field effect transistors (TEFTs) ${ }^{4}$ or Esaki diodes, ${ }^{5}$ or infra-red laser and detectors, ${ }^{6,7}$ and photovoltaic detectors ${ }^{8}$ has been reported in the literature, the manipulation of optical polarization anisotropy in these NWs, which has diverse applications, e.g., in manipulating the signal bandwidth in optoelectronic circuits, is still unexplored. ${ }^{3}$

In this article, we compare the wavelength sensitivity of the polarization anisotropy of Raman intensity in single bare InAs and InAs/GaSb core-shell NWs. We show that the polarization anisotropy has a non-linear variation with the excitation wavelength in InAs/GaSb core-shell NWs. Our experimental findings are explained by a model calculation of the value of $\rho$ using the combined contributions of the Raman tensor components and stored electromagnetic energy inside bare InAs and InAs/GaSb core-shell NWs. This work focuses on the role of dielectric mismatch of the constituents in deciding the above characteristics of the core-shell NWs. In addition, six-fold enhancement of the scattering intensity of InAs/GaSb core-shell NWs with respect to that of bare InAs NWs has been observed.

\footnotetext{
${ }^{a)}$ Email: anushree@phy.iitkgp.ernet.in

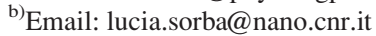

Bare InAs NWs (NW1) and InAs/GaSb NWs (NW2) were grown on $\mathrm{Si}$ (111) substrates using a chemical beam epitaxy technique with a catalyst-free approach. ${ }^{9}$ The growth was started with a nucleation step at $420 \pm 10^{\circ} \mathrm{C}$ for $5 \mathrm{~min}$, using metal organic line pressures of 0.3 and 3.0 Torr for trimethylindium (TMIn) and tertiarybutylarsine (TBAs), respectively. This step was followed by a $10 \mathrm{~min}$ ramp to the growth temperature of $520 \pm 10^{\circ} \mathrm{C}$. For NW1, the growth was continued at this temperature for $60 \mathrm{~min}$, using 0.20 and 3.00 Torr of TMIn and TBAs, respectively. For InAs/GaSb core-shell NWs (NW2), first, the core was grown following the same growth protocol as followed in the case of NW1. Next, the GaSb shell was grown for $30 \mathrm{~min}$ at the same substrate temperature, using triethylgallium (TEGa) at a pressure of 0.70 Torr and trimethylantimony (TMSb) at a pressure of 0.60 Torr. The details of the growth procedure are available in Refs. 5 and 9. For Raman measurements, the NWs were transferred on the HF cleaned $\mathrm{Si}$ wafer by dispersing the NWs in a liquid solvent and following a drop-casting technique. Micro-Raman spectra of single NWs in the backscattering configuration were recorded using a triple monochromator (model T64000, JY, France). The laser power was kept at $\sim 30 \mu \mathrm{W}$ on the sample to avoid the effect of local laser heating. ${ }^{10}$ Polarization dependent Raman measurements were carried out using $\lambda / 2$ plates in the path of the incident and scattered lights. Discrete laser lines of the $\mathrm{Ar}^{+}-\mathrm{Kr}^{+}$laser (Inova70C, Coherent USA) were chosen as excitation energies.

The NWs were additionally characterized by scanning electron microscopy (SEM). The SEM images of NW1 and NW2 are shown in Figs. 1(a) and 1(b), respectively. The inset of Fig. 1(b) shows the top view of a core-shell NW. The average lengths of NW1 and NW2 are $0.75 \pm 0.1 \mu \mathrm{m}$ and 0.80 $\pm 0.1 \mu \mathrm{m}$, respectively, while the corresponding diameters are $50 \pm 10 \mathrm{~nm}$ for NW1 and $120 \pm 10 \mathrm{~nm}$ for NW2 with a shell thickness of $35 \pm 10 \mathrm{~nm}$. The NWs contain both zincblende 


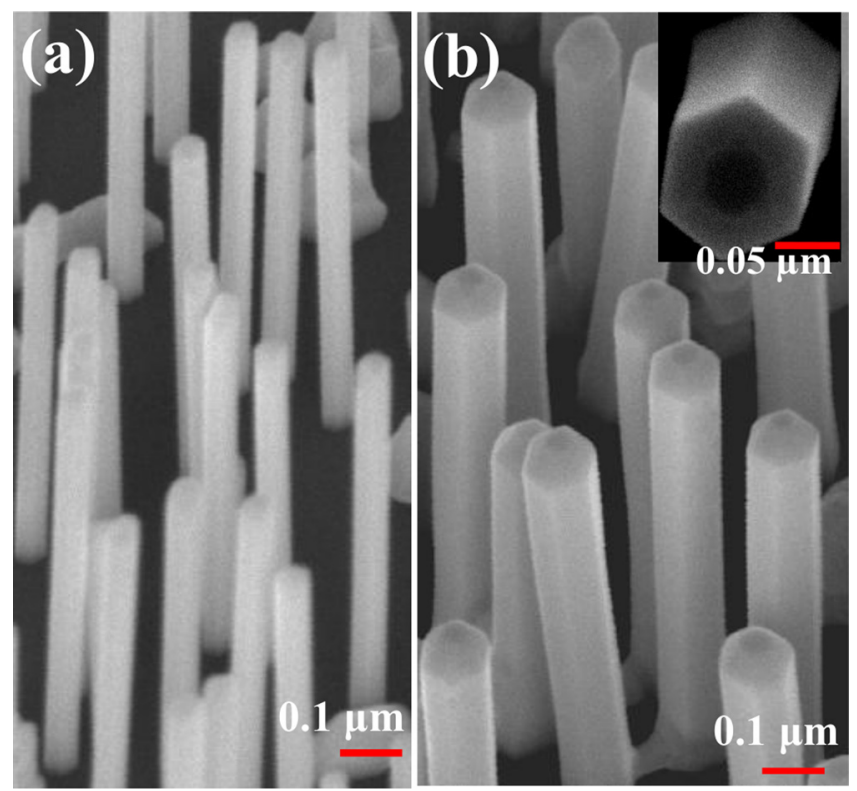

FIG. 1. SEM image of (a) bare InAs NWs and (b) InAs/GaSb core-shell NWs. The top view of a characteristic core-shell NW is shown in the inset of (b). The colour contrast depicts the core and shell regions.

(ZB) and wurtzite (WZ) phases. The details of crystallinity, phase, and elemental analysis of these NWs, as obtained from high resolution TEM measurements and energy dispersive $\mathrm{x}$-ray spectroscopy, have been published elsewhere. ${ }^{5,9}$

Unpolarized Raman spectra of individual NWs from NW1 and NW2 samples over the spectral range between $180 \mathrm{~cm}^{-1}$ and $270 \mathrm{~cm}^{-1}$ were recorded using the laser excitation wavelength as $514.5 \mathrm{~nm}$ and are shown in Figs. 2(a) and 2(b), respectively. In the figure, + symbols are experimental data points. Typical Raman spectra of InAs and GaSb in ZB and WZ phases over the above spectral range exhibit transverse optical (TO) and longitudinal optical (LO) modes. Due to the folding of the Brillouin zone, for the WZ phase of the same compounds, one observes an additional Raman mode $\left(\mathrm{E}^{2 \mathrm{H}}\right.$ mode) below the TO mode. Since the bare InAs NW has a mixed phase, the Raman spectrum is expected to exhibit all the above-mentioned Raman modes. Thus, the Raman spectrum of NW1 in Fig. 2(a) is deconvoluted with three Lorentzian functions (LO and TO modes originating from both WZ- and ZB-InAs along with the $\mathrm{E}^{2 \mathrm{H}}$ mode due to WZ-InAs only), keeping intensities of all modes as free fitting parameters. As the $\mathrm{TO}$ and $\mathrm{E}^{2 \mathrm{H}}$ modes are not resolved, their peak positions were allowed to vary within $5 \%$ of their expected values. ${ }^{11}$ In Fig. 2(a), the deconvoluted components are shown by dashed lines. The TO and LO modes of NW1 appear at 217 and $238 \mathrm{~cm}^{-1}$, respectively (green dashed line), while the additional $\mathrm{E}^{2 \mathrm{H}}$ mode of WZInAs appears at $214 \mathrm{~cm}^{-1}$ (black dashed line). The overall fit to the experimental data points, which is the sum of the above components, is shown as the red solid line.

Since the core and the shell of sample NW2 are also of mixed phases, the Raman spectrum of NW2 is expected to exhibit the following components: (i) LO and TO modes of ZB- and WZ-InAs, (ii) TO and LO modes of WZ- and ZB$\mathrm{GaSb}$, (iii) the $\mathrm{E}^{2 \mathrm{H}}$ mode of WZ-GaSb, and (iv) the $\mathrm{E}^{2 \mathrm{H}}$ mode of WZ-InAs. The $\mathrm{E}^{2 \mathrm{H}}$ modes of GaSb and InAs are

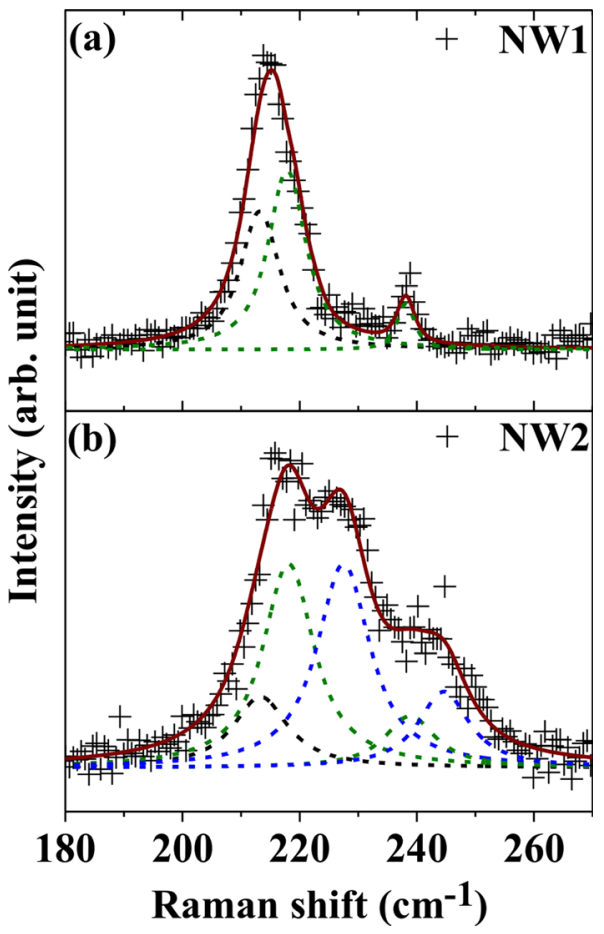

FIG. 2. The unpolarized Raman spectrum of (a) single bare InAs and (b) InAs/GaSb NWs with $514 \mathrm{~nm}$ as the excitation wavelength. + symbols are experimental data points. Deconvoluted LO-TO modes of InAs and GaSb are shown by green and blue dashed lines, respectively. The black dashed lines correspond to the $\mathrm{E}^{2 \mathrm{H}}$ mode of WZ-InAs [in (a)] and to overlapping $\mathrm{E}^{2 \mathrm{H}}$ modes of WZ-GaSb and WZ-InAs [in (b)]. The net fitted spectra are shown by red solid lines.

expected to appear at 212 and $214 \mathrm{~cm}^{-1}$. Thus, it is nontrivial to resolve these two modes at room temperature. Hence, the Raman spectrum of NW2 is deconvoluted with five Lorentzian functions, keeping intensities as free parameters. As the peaks are not well-resolved, all peak positions were allowed to vary within $5 \%$ of their expected values. ${ }^{11,12}$ The widths were kept nearly the same for all modes. For NW2, the ZB- and WZ-InAs-like TO and LO modes appear at 218 and $238 \mathrm{~cm}^{-1}$, respectively. The peak at $213 \mathrm{~cm}^{-1}$ appears due to overlapped $\mathrm{E}^{2 \mathrm{H}}$ modes of WZ-InAs and WZGaSb. The TO and LO modes for WZ- and ZB-GaSb shell components appear at 227 and $244 \mathrm{~cm}^{-1}$, respectively. Deconvoluted WZ/ZB InAs and GaSb spectral components are shown by green and blue dashed lines in Fig. 2(b), respectively. The deconvoluted $\mathrm{E}^{2 \mathrm{H}}$ component (of both WZ-InAs and WZ-GaSb) is shown by the black dashed line. The net fitted spectrum is shown as the red solid line.

We carried out polarization dependent Raman measurements for different excitation energies on NW1 and NW2. Raman spectra were deconvoluted following the procedure adopted and discussed in Fig. 2. For our studies, we have considered the integral intensity of the most prominent InAs TO phonon mode of the NWs for different angles $(\phi)$ between the polarization direction of the excitation radiation and the axis of the NW (see the schematic diagram on top in Fig. 3). The azimuthal dependence of the Raman intensity of the InAs TO mode for NW2 for different incident excitation wavelengths is shown in Fig. 3, while the polar plots for NW1 are available in Fig. S1 in the supplementary material. In these figures, the distance of the data points from the center of the circle 


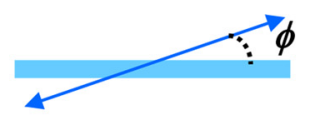

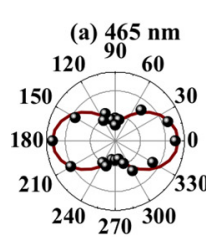

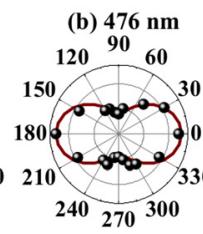

(g) $520 \mathrm{~nm}$
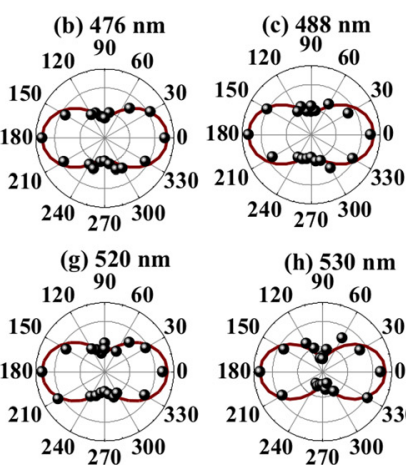

(h) $530 \mathrm{~nm}$
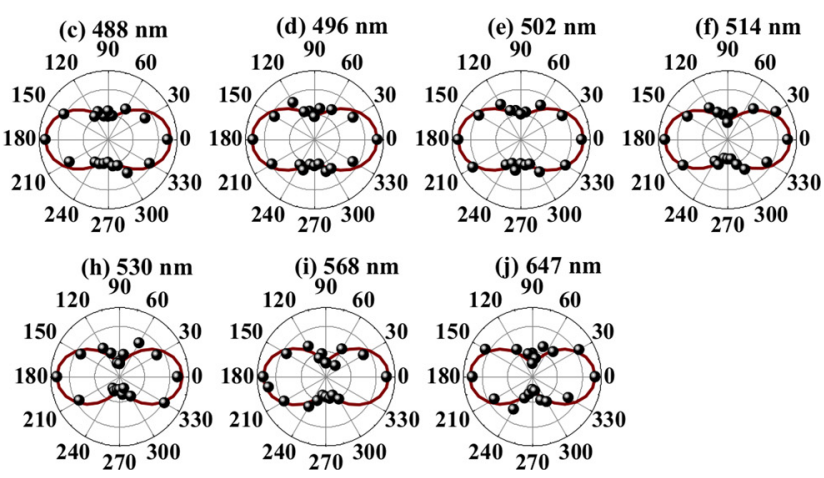

(j) $647 \mathrm{~nm}$

$120^{90} 60$

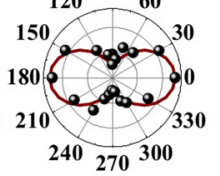

FIG. 3. Polar plots of the Raman intensity of the InAs TO mode for different excitation wavelengths for NW2. Top: Schematic configuration followed to carry out the polarization dependent Raman measurements. Arrow marks the direction of polarization with respect to the axis of the NW (blue bar). corresponds to the intensity scale of the Raman intensity of the InAs TO mode. For example, the Raman intensity is maximum for $\phi=0$ or $360^{\circ}$. We find that for NW2, the ratio of maximum and minimum intensity $\left(I_{N W}^{\|}\right.$and $\left.I_{N W}^{\perp}\right)$ varies nonmonotonically with the increase in the excitation wavelength. $I_{N W}^{\|}$and $I_{N W}^{\perp}$ derived from the polar plots, observed in two orthogonal polarization configurations, can be correlated with the polarization anisotropy of the scattered light from the NWs. The variation of the observed value of the polarization anisotropy, $\rho=\left(I_{N W}^{\|}-I_{N W}^{\perp}\right) /\left(I_{N W}^{\|}+I_{N W}^{\perp}\right)$, with laser excitation wavelengths for NW1 and NW2, is shown by symbols in Figs. 4(a) and 4(b), respectively. The measurements have been repeated on 5 individual NWs to check the reproducibility of the results. The experimental data points and the error bars in Fig. 4 are the average values and the corresponding standard deviations of $\rho$ obtained from measurements on 5 different NWs of each sample. We find that the value of $\rho$ increases from $0.63 \pm 0.04$ to $0.76 \pm 0.05$ for NW1 [Fig. 4(a)], while it varies non-monotonically over the given range of excitation wavelengths for NW2 [Fig. 4(b)]. In particular, $\rho$ for NW2 has a dip from $0.55 \pm 0.05$ to $0.45 \pm 0.03$ between

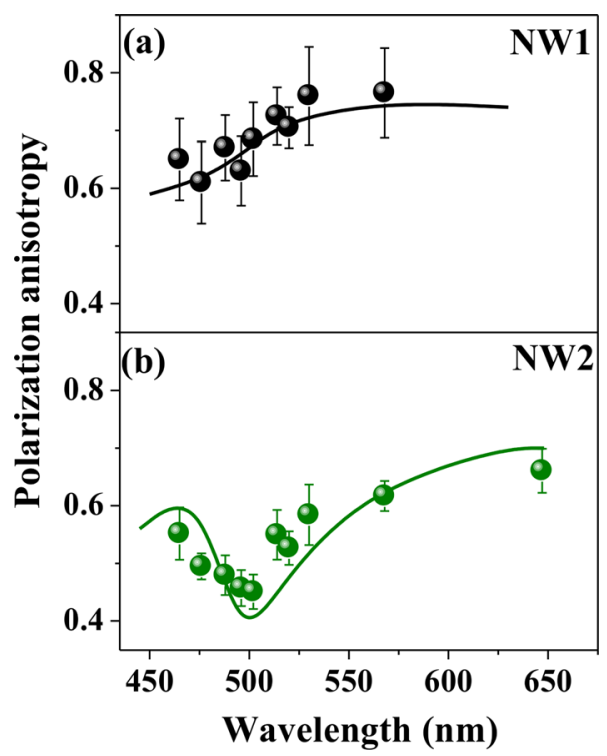

FIG. 4. The polarization anisotropy of (a) bare InAs NWs (NW1) and (b) InAs/GaSb core shell NWs (NW2): Solid lines correspond to the calculated values using Eq. (1), while the symbols are the experimentally obtained values of $\rho$, from the plots in Figs. 3 and S1 (supplementary material).
465 and $502 \mathrm{~nm}$ and rises again for higher excitation wavelengths.

In order to have a better understanding of the above results, we carry out a model calculation. The Raman scattering intensity of a NW can be written as ${ }^{13} I_{s} \propto \omega_{i}^{4}\left|\hat{e}_{s} \cdot \tilde{Q} \cdot \Re \cdot \tilde{Q} \cdot \hat{e}_{i}\right|^{2} I_{0}$, where $\hat{e}_{i}$ and $\hat{e}_{s}$ are the directions of polarization of the incident and the scattered lights and $\Re$ is the Raman tensor representing the symmetry of the Raman active phonon modes. $\omega_{i}$ and $I_{O}$ are the frequency and the intensity of the incident radiation, respectively. The tensor $\tilde{Q}$ describes the enhancement of radiation due to the confinement of the photon within the NW. The non-zero components $Q_{\|}$and $Q_{\perp}$ define the enhancement of the electric field inside the NW when the incident polarization is parallel and perpendicular to the NW axis, respectively. In terms of $Q_{\|}$ and $Q_{\perp}$, the Raman scattering intensity for a single NW can be written as ${ }^{13}$

$$
\begin{gathered}
I \propto\left(R_{22} Q_{\|}^{2} \cos ^{2} \phi+R_{23} Q_{\|} Q_{\perp} \cos \phi \sin \phi\right. \\
\left.+R_{32} Q_{\|} Q_{\perp} \cos \phi \sin \phi+R_{33} Q_{\perp}^{2} \sin ^{2} \phi\right)^{2} .
\end{gathered}
$$

As mentioned earlier, the angle between the incident polarization and the NW axis is defined by $\phi$. The values of Raman tensor components, $R_{i j}$, depend on the growth direction of the NW. Considering the NW as an infinite cylinder, an approximation quite reasonable due to the small aspect ratio of about 0.06 for both NW1 and NW2, for a polarized EM radiation of wavelength $\lambda$, one solves Maxwell's equation of a scalar function in cylindrical polar coordinates to obtain the internal and scattered fields. The parallel and perpendicular electric field enhancement factors $Q_{\|}$and $Q_{\perp}$ in an infinite cylinder of radius $a$ and dielectric constant $\varepsilon_{2}$ surrounded by the shell of radius $b$ and dielectric constant $\varepsilon_{1}$ can be expressed as ${ }^{14,15}$

$$
\mathrm{Q}_{\|}=E_{0}^{2} \sum_{\mathrm{n}=-\infty}^{\infty}\left|f_{\mathrm{n}}\right|^{2}\left[\mathrm{~J}_{\mathrm{n}}^{2}\left(\varepsilon_{2} k_{0} a\right)-\mathrm{J}_{\mathrm{n}-1}\left(\varepsilon_{2} k_{0} a\right) \mathrm{J}_{\mathrm{n}+1}\left(\varepsilon_{2} k_{0} a\right)\right] / \mathrm{I}_{\mathrm{bulk}}
$$

and

$$
\begin{aligned}
\mathrm{Q}_{\perp}= & \frac{E_{0}^{2}}{2} \sum_{\mathrm{n}=-\infty}^{\infty}\left|g_{\mathrm{n}}\right|^{2}\left[\mathrm{~J}_{\mathrm{n}-1}^{2}\left(\varepsilon_{2} k_{0} a\right)+\mathrm{J}_{\mathrm{n}+1}^{2}\left(\varepsilon_{2} k_{0} a\right)\right. \\
& \left.-\mathrm{J}_{\mathrm{n}}\left(\varepsilon_{2} k_{0} a\right)\left\{\mathrm{J}_{\mathrm{n}-2}\left(\varepsilon_{2} k_{0} a\right)+\mathrm{J}_{\mathrm{n}+2}\left(\varepsilon_{2} k_{0} a\right)\right\}\right] / \mathrm{I}_{\text {bulk }} .
\end{aligned}
$$


Here, $E_{0}$ is the electric field of the incident radiation, $I_{\text {bulk }}$ is the average intensity in the bulk dispersive medium, while $f_{n}$ and $g_{n}$ are related to far field scattering coefficients and are functions of $a, b, \varepsilon_{1}, \varepsilon_{2}$, and $\varepsilon_{m}$ (see supplementary material, $\mathrm{S} 2)$. It is to be noted that the values of $\varepsilon_{1}$ and $\varepsilon_{2}$ depend on the incident excitation wavelength, and $k_{0}$ is the propagation constant of the incident light in vacuum. $J_{n}\left(\varepsilon_{2} k_{0} a\right)$ and $H_{n}\left(\varepsilon_{2} k_{0} a\right)$ are Bessel and Henkel functions of the first kind, respectively. $\varepsilon_{\mathrm{m}}$ is the dielectric constant of the surrounding medium. For a bare NW, the expression of $\mathrm{Q}_{\|}$and $\mathrm{Q}_{\perp}$ can be obtained by considering $b=a$ and $\varepsilon_{1}=\varepsilon_{2}$ in $f_{\mathrm{n}}$ and $g_{\mathrm{n}}$. Following Eqs. (1)-(3), Raman intensity from a single NW is expected to vary with the angle $\phi$ and modulated by the excitation wavelength. By using Eqs. (1)-(3), the variation of $\rho$ for different excitation wavelengths for both NW1 and NW2 has been calculated and are shown by the solid lines in Fig. 4. Since the dielectric constants of WZ-InAs and WZ$\mathrm{GaSb}$ are not available for all excitation wavelengths of interest, in this calculation, we have only used the values of $\varepsilon_{1}$ and $\varepsilon_{2}$ for ZB-GaSb and ZB-InAs from Ref. 16 for both $\mathrm{ZB}$ and $\mathrm{WZ}$ phase components. However, the Raman tensor components $R_{22}$ and $R_{33}$ in Eq. (1) for $\mathrm{ZB}$ and $\mathrm{WZ}$ phases are obtained from Ref. 13. As the crystal structure of NW1 and NW2 has both $\mathrm{ZB}$ and WZ phases along the length, to take into account the difference in the scattering efficiency of these phases, in the simulation, we considered the scattering intensity of $\mathrm{ZB}$ and $\mathrm{WZ}$ components with different weight percents. For NW1, the weight factor of $\mathrm{WZ}$ and $\mathrm{ZB}$ components as 20:80 matched the experimental data fairly well. For the core-shell NWs, the green solid line in Fig. 4 corresponds to the weight factor of the $\mathrm{WZ}$ and $\mathrm{ZB}$ ratio as 10:90. The calculated plots for NW1 and NW2 are shifted by $10 \mathrm{~nm}$ and $15 \mathrm{~nm}$ towards the lower wavelength to match the experimentally observed values of $\rho$. The origin of this required shift of the theoretical plots may be the fact that the wavelength dependent dielectric constants of both $\mathrm{ZB}$ and WZ phases are considered to be the same in our model, while the optical properties of non-nitride III-V semiconductor compounds are expected to slightly differ. ${ }^{17}$ Our results indicate that the polarisation anisotropy in InAs/GaSb NWs can be tuned by selecting the core/shell diameter and the excitation wavelength. It is also important to note that its value is less than what one find in bare InAs NWs.

Furthermore, we demonstrate the enhancement of Raman scattering intensity due to the addition of the $\mathrm{GaSb}$ shell around the NWs. We consider the Raman intensity of the InAs TO mode from individual NW1 and NW2 in the $z(x x) \bar{z}$ scattering configuration for different excitation wavelengths. Note that in this polarization configuration, the Raman intensity is dominated by the $\mathrm{ZB}$ phase of the NWs. ${ }^{18}$ We estimate the enhancement in scattering intensity as $S 1=\left(I_{N W 2}^{o b s} / V_{N W 2}\right) /\left(I_{N W 1}^{o b s} / V_{N W 1}\right)$ (see Fig. S2 in the supplementary material). $I_{N W 1}^{o b s}$ and $I_{N W 2}^{o b s}$ are the measured intensities of the most intense InAs-TO mode of single NW1 and NW2, respectively, and $V_{N W 1}$ and $V_{N W 2}$ are the probed volumes of NW1 and the NW2, respectively. We find that Raman scattering is enhanced $(S 1>1)$ by the presence of the shell for excitation wavelengths in the range of 480-570 nm. Most importantly, the enhancement can reach a peak value of $6.0 \pm 0.5$ at the excitation wavelength of $\sim 514 \mathrm{~nm}$. We also estimate the enhancement in the scattering intensity of NW2 with respect to bulk InAs as $S 2=\left(I_{N W 2}^{o b s} / V_{N W 2}\right) /$ $\left(I_{\text {bulk }} / V_{\text {bulk }}\right)$ and show in the inset of Fig. S2 in the supplementary material. $I_{b u l k}$ is the measured intensities of the TO mode of bulk InAs. $V_{\text {bulk }}$ is the probed volume of the bulk. We experimentally find nearly 1000 times increase in the intensity of the scattered light for $520 \mathrm{~nm}$ as the excitation wavelength (see the inset of Fig. S2 in the supplementary material). A similar enhancement in scattering intensity has been reported earlier for InAs NWs ${ }^{19}$ and Si nanocones. ${ }^{14}$

In summary, we have investigated the effect of the shell in governing the non-linear polarization anisotropy of the Raman scattering intensity in InAs/GaSb core-shell NWs with respect to bare InAs NWs. The observed behaviour of the core-shell NWs could be explained by considering the confinement of electromagnetic radiation within the NW and Raman tensor components at different polarization geometries. Control over the degree of polarization anisotropy is crucial for the employment of these nanostructures in photonic devices. By selecting appropriate growth parameters for fabricating NWs with the desired diameter, core/shell materials, and the excitation wavelength, the polarization anisotropy can be suitably tailored. Furthermore, we have shown that in the InAs/GaSb NW, the shell plays an important role in enhancing the intensity of Raman scattering.

See supplementary material for the polar plots of the Raman intensity of the InAs TO mode for varying excitation wavelengths for NW1 at different wavelengths. The full expressions for $f_{n}$ and $g_{n}$ in Eqs. (2) and (3) are available in $\mathrm{S} 2$. The measured enhancement in the intensity of NW2 with respect to NW1 and bulk InAs is shown in S3.

A.R. thanks the Department of Science and Technology, Government of India, for financial assistance.

${ }^{1}$ B. Mayer, D. Rudolph, J. Schnell, S. Morkötter, J. Winner, J. Treu, K. Müller, G. Bracher, G. Abstreiter, G. Koblmüller, and J. J. Finley, Nat. Commun. 4, 2931 (2013).

${ }^{2}$ H. J. Joyce, P. Parkinson, N. Jiang, C. J. Docherty, Q. Gao, H. H. Tan, C. Jagadish, L. M. Herz, and M. B. Johnston, Nano. Lett. 14, 5989 (2014).

${ }^{3}$ J. F. Wang, M. S. Gudiksen, X. F. Duan, Y. Cui, and C. M. Lieber, Science 293, 1455 (2001).

${ }^{4}$ J. Knoch and J. Appenzeller, J. IEEE Electron Device Lett. 31, 305 (2010).

${ }^{5}$ M. Rocci, F. Rossella, U. P. Gomes, V. Zannier, F. Rossi, D. Ercolani, L. Sorba, F. Beltram, and S. Roddaro, Nano. Lett. 16, 7950 (2016).

${ }^{6}$ B. V. Olson, L. M. Murray, J. P. Prineas, M. E. Flatté, J. T. Olesberg, and T. F. Boggess, Appl. Phy. Lett. 102, 202101 (2013).

${ }^{7}$ N. Gautam, H. S. Kim, M. N. Kutty, E. Plis, L. R. Dawson, and S. Krishna, Appl. Phys. Lett. 96, 231107 (2010).

${ }^{8}$ Y. Wei, A. Gin, M. Razeghi, and G. J. Brown, Appl. Phys. Lett. 81, 3675 (2002).

${ }^{9}$ U. P. Gomes, D. Ercolani, N. V. Sibirev, M. Gemmi, V. G. Dubrovskii, F. Beltram, and L. Sorba, Nanotechnology 26, 415604 (2015).

${ }^{10}$ A. Patra, J. K. Panda, A. Roy, M. Gemmi, J. David, D. Ercolani, and L. Sorba, Appl. Phy. Lett. 107, 093103 (2015).

${ }^{11}$ N. G. Hörmann, I. Zardo, S. Hertenberger, S. Funk, S. Bolte, M. Doblinger, G. Koblmüller, and G. Abstreiter, Phys. Rev. B 84, 155301 (2011).

${ }^{12}$ K. Aoki, E. Anastassakis, and M. Cardona, Phys. Rev. B. 30, 681 (1984). 
${ }^{13}$ J. Wu, D. Zhang, Q. Lu, H. R. Gutierrez, and P. C. Eklund, Phys. Rev. B 81, 165415 (2010).

${ }^{14}$ L. Cao, B. Nabet, and J. E. Spanier, Phys. Rev. Lett. 96, 157402 (2006).

${ }^{15}$ J. Giblin, V. Protasenko, and M. Kuno, ACS Nano 3, 1979 (2009).

${ }^{16}$ E. D. Palik, Handbook of Optical Constants of Solid (Academic Press London, 1985), pp. 479-597.
${ }^{17}$ A. De and C. E. Pryor, Phys. Rev. B 85, 125201 (2012).

${ }^{18}$ M. Möller, M. M. Lima, A. Cantarero, L. C. O. Dacal, J. R. Madureira, F. Iikawa, T. Chiaramonte, and M. A. Cotta, Phys. Rev. B 84, 085318 (2011).

${ }^{19}$ J. K. Panda, A. Roy, A. Singha, M. Gemmi, D. Ercolani, V. Pellegrini, and L. Sorba, Solid State Commun. 160, 26 (2013). 